TITLE:

\title{
Variable anisotropy of ionic conduction in lithium nitride: Effect of duplex-charge transfer
}

\section{AUTHOR(S):}

Kishida, Ippei; Oba, Fumiyasu; Koyama, Yukinori; Kuwabara, Akihide; Tanaka, Isao

\section{CITATION:}

Kishida, Ippei ...[et al]. Variable anisotropy of ionic conduction in lithium nitride: Effect of duplex-charge transfer. PHYSICAL REVIEW B 2009, 80(2): 024116.

\section{ISSUE DATE:}

2009-06

URL:

http://hdl.handle.net/2433/109876

\section{RIGHT:}

(c) 2009 The American Physical Society 
PHYSICAL REVIEW B 80, 024116 (2009)

\title{
Variable anisotropy of ionic conduction in lithium nitride: Effect of duplex-charge transfer
}

\author{
Ippei Kishida, ${ }^{*}$ Fumiyasu Oba ${ }^{\dagger}$ and Yukinori Koyama \\ Department of Materials Science and Engineering, Kyoto University, Sakyo, Kyoto 606-8501, Japan
}

Akihide Kuwabara

Nanostructures Research Laboratory, Japan Fine Ceramics Center, Atsuta, Nagoya 456-8587, Japan

Isao Tanaka

Department of Materials Science and Engineering, Kyoto University, Sakyo, Kyoto 606-8501, Japan

and Nanostructures Research Laboratory, Japan Fine Ceramics Center, Atsuta, Nagoya 456-8587, Japan

(Received 5 September 2008; revised manuscript received 5 June 2009; published 27 July 2009)

\begin{abstract}
The formation and migration of defects relevant to the $\mathrm{Li}$ ionic conduction in $\mathrm{Li}_{3} \mathrm{~N}$ have been investigated using first-principles calculations. For undoped $\mathrm{Li}_{3} \mathrm{~N}$, Frenkel reactions generating the Li vacancy and two types of $\mathrm{Li}$ interstitial are found to dominate the defect equilibria. In the layered structure of $\mathrm{Li}_{3} \mathrm{~N}$, the $\mathrm{Li}$ vacancy migrates selectively toward the intralayer direction, whereas the Li interstitial readily moves in both intralayer and interlayer directions. Despite the significant crystallographic anisotropy and the orientation dependence of dominant charge carriers, the resultant activation energy for the Li ionic conduction is nearly isotropic in the undoped system. The presence of $\mathrm{H}$ impurities yields $\mathrm{Li}$ vacancy-rich defect equilibria, leading to an anisotropic ionic conduction governed by the Li vacancy. These findings elucidate the variable anisotropy in the ionic conductivity of $\mathrm{Li}_{3} \mathrm{~N}$.
\end{abstract}

DOI: 10.1103/PhysRevB.80.024116

PACS number(s): 66.30.hd, 61.72.Bb, 61.72.J-

\section{INTRODUCTION}

Because of the demands for further downscaling and lightweighting of portable electronic devices and for wider applications of batteries, the development of highperformance electrolytes and electrodes has been a key issue in lithium-ion battery technologies. Lithium nitride $\left(\mathrm{Li}_{3} \mathrm{~N}\right)$, a prototype of the fast solid-state ionic conductor, has motivated fundamental studies on its superb $\mathrm{Li}$ ionic conductivity $^{1-15}$ and the exploration of related novel ionic/ mixed conductors for electrolyte and electrode applications. ${ }^{16-19}$ The layered structure of $\mathrm{Li}_{3} \mathrm{~N}$ leads to an anisotropic Li ionic conduction, where the intralayer conductivity is greater than the interlayer conductivity. Interestingly, the magnitude of the anisotropy depends on the type of specimen, ${ }^{4,6-8}$ which cannot be explained by a simple mechanism such as an orientation-dependent migration energy of a dominant charge carrier species. It was suggested that this behavior is related to hydrogen $(\mathrm{H})$ impurities intentionally or unintentionally incorporated. ${ }^{6,7} \mathrm{Wahl}^{6}$ reported that the anisotropy is small in undoped specimens, and it increases significantly with $\mathrm{H}$ doping. The microscopic mechanism behind the variable anisotropy, however, has not been established.

In previous works on the $\mathrm{Li}$ conduction/diffusion in $\mathrm{Li}_{3} \mathrm{~N}$, it was considered that the $\mathrm{Li}$ vacancy $\left(V_{\mathrm{Li}}\right)$ is the dominant charge carrier. The migration mechanism of $V_{\mathrm{Li}}$ has been proposed by Sarnthein et al. ${ }^{14}$ through first-principles molecular dynamics calculations. Wolf et al. ${ }^{12,13}$ has pointed out on the basis of molecular dynamics simulations using interatomic potentials that the $\mathrm{Li}$ interstitial $\left(\mathrm{Li}_{\mathrm{i}}\right)$ also contributes to the $\mathrm{Li}$ ionic conduction/diffusion at high temperatures. However, the structure and migration mechanism of $\mathrm{Li}_{\mathrm{i}}$ have not been clarified at an atomistic level. The detailed understanding of the outstanding ionic conductivity of $\mathrm{Li}_{3} \mathrm{~N}$ is im- portant since it is expected to provide a useful guideline for the design and exploration of novel high-performance ionic conductors.

In the present study, we revisit the Li ionic conduction mechanism in $\mathrm{Li}_{3} \mathrm{~N}$ using extensive first-principles calculations on the formation and migration of $V_{\mathrm{Li}}$ and $\mathrm{Li}_{\mathrm{i}}$, including the effect of the $\mathrm{H}$ impurity. It is found that $\mathrm{Li}_{3} \mathrm{~N}$ has duplexcharge carriers: not only $V_{\mathrm{Li}}$ but also $\mathrm{Li}_{\mathrm{i}}$ plays an essential role, depending on the crystallographic orientation and the presence and absence of $\mathrm{H}$ impurities. The variable anisotropic $\mathrm{Li}$ ionic conduction in $\mathrm{Li}_{3} \mathrm{~N}$ can be understood from the formation and migration of the duplex carriers.

\section{COMPUTATIONAL METHODS}

The calculations were performed using density functional theory ${ }^{20,21}$ with the plane-wave projector augmented-wave (PAW) method ${ }^{22}$ as implemented in the VASP code. ${ }^{23-25}$ The exchange-correlation term was treated with the PerdewBurke-Ernzerhof functional ${ }^{26}$ based on the generalized gradient approximation. PAW data sets having radial cutoffs of $1.1,0.8$, and $0.6 \AA$ for $\mathrm{Li}, \mathrm{N}$, and $\mathrm{H}$, respectively, and a plane-wave cutoff energy of $400 \mathrm{eV}$ were employed. For defect calculations, supercells containing 300 atoms were constructed by the $5 \times 5 \times 3$ expansion of the $\mathrm{Li}_{3} \mathrm{~N}$ unit cell. $k$-point sampling was conducted only at the $\Gamma$ point since the test calculations for major defects, i.e., $V_{\mathrm{Li}}$ and $\mathrm{Li}_{\mathrm{i}}$, using a $2 \times 2 \times 2 k$-point mesh indicated the convergence of the formation energies within $0.01 \mathrm{eV}$. The ionic positions were relaxed until the residual forces became less than $0.01 \mathrm{eV} / \AA$, with the lattice constants fixed at the values optimized for the perfect crystal: $a=3.651$ and $c=3.888 \AA$, which overestimate experimental values of $a=3.641$ and $c$ $=3.872 \AA$ (Ref. 27 ) by $0.3 \%$ and $0.4 \%$, respectively. 
To discuss the energetics for the $\mathrm{Li}$ ionic conduction, defect formation and migration were separately studied. Concerning the former, the formation energies of native defects and $\mathrm{H}$ impurities in relevant charge states were evaluated as $^{28,29}$

$$
E^{\mathrm{f}}=E_{\mathrm{tot}}^{\mathrm{def}}-E_{\mathrm{tot}}^{\mathrm{per}}-\sum_{i} \Delta n_{i} \mu_{i}+q E_{\mathrm{F}},
$$

where $E_{\mathrm{tot}}^{\mathrm{def}}$ and $E_{\mathrm{tot}}^{\mathrm{per}}$ denote the total energy of the supercell containing a defect in charge state $q$ and that of the perfectcrystal supercell, respectively. $\Delta n_{i}$ is the difference in the number of constituent atom $i$ between these supercells. $\mu_{i}$ $(i=\mathrm{Li}, \mathrm{N}$, and $\mathrm{H})$ denotes the atomic chemical potentials and $E_{\mathrm{F}}$ is the Fermi level. The reference of $E_{\mathrm{F}}$ for the defect supercells was aligned with that for the perfect crystal using the average electrostatic potentials at ionic positions farthest from the defect and that in the perfect crystal. ${ }^{28-31}$

The chemical potentials of $\mathrm{Li}$ and $\mathrm{N}$ that constitute host $\mathrm{Li}_{3} \mathrm{~N}$ can vary under a correlation of

$$
3 \mu_{\mathrm{Li}}+\mu_{\mathrm{N}}=\mu_{\mathrm{Li}_{3} \mathrm{~N}(\mathrm{bulk})},
$$

where $\mu_{\mathrm{Li}_{3} \mathrm{~N}(\mathrm{bulk})}$ is the chemical potential of bulk $\mathrm{Li}_{3} \mathrm{~N}$. The upper limits of $\mu_{\mathrm{Li}}$ and $\mu_{\mathrm{N}}$ were set at $\mu_{\mathrm{Li}}=\mu_{\mathrm{Li} \text { (bulk) }}$ (Li rich)

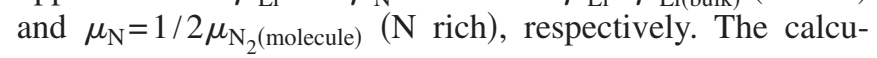
lated total energies per unit formula were used for the chemical potentials of the reference systems. The upper limit of $\mu_{\mathrm{H}}$, i.e., the $\mathrm{H}$-rich limit, was determined assuming the equilibria of $\mathrm{H}$-doped $\mathrm{Li}_{3} \mathrm{~N}$ with relevant $\mathrm{Li}-\mathrm{H}-\mathrm{N}$ compounds; those showing negative formation energies, which are $\mathrm{LiNH}_{2}$ and $\mathrm{LiH}$ among $\mathrm{Li}_{4} \mathrm{NH}, \mathrm{Li}_{2} \mathrm{NH}, \mathrm{LiNH}_{2}$, and $\mathrm{LiH}$, were considered. The equilibrium among $\mathrm{Li}_{3} \mathrm{~N}, \mathrm{LiNH}_{2}$, and $\mathrm{LiH}$ was found to yield the highest value of $\mu_{\mathrm{H}}$, which is given, in addition to Eq. (2), as

$$
\begin{gathered}
\mu_{\mathrm{Li}}+\mu_{\mathrm{N}}+2 \mu_{\mathrm{H}}=\mu_{\mathrm{LiNH}_{2}(\text { bulk })}, \\
\mu_{\mathrm{Li}}+\mu_{\mathrm{H}}=\mu_{\mathrm{LiH}(\text { bulk })} .
\end{gathered}
$$

We consider this $\mathrm{H}$-rich limit as an extreme case of $\mathrm{H}$-doped $\mathrm{Li}_{3} \mathrm{~N}$.

Assuming a dilute regime in which the interactions between defects can be neglected, the equilibrium concentration of defect $j$ is estimated as ${ }^{29,32}$

$$
C_{j}=N_{j} \exp \left(-\frac{E_{j}^{\mathrm{f}}}{k_{\mathrm{B}} T}\right),
$$

where $N_{j}$ denotes the number of sites for defect $j$. In the case of charged defects, for which formation energies depend on the Fermi level as given in Eq. (1), the equilibrium concentrations are determined via the charge neutrality condition. Fully ionic charge compensation can be reasonably assumed for $\mathrm{Li}_{3} \mathrm{~N}$ exhibiting essentially pure ionic conductivity. Therefore, the contributions of electrons and holes were neglected, and only fully charged defects were considered. The charge neutrality condition is then given as (a)

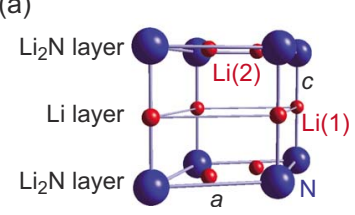

(b)

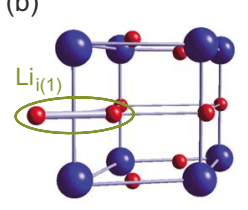

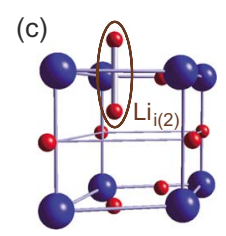

FIG. 1. (Color online) (a) Unit cell of $\mathrm{Li}_{3} \mathrm{~N}$ (space group: $P 6 / \mathrm{mmm}$ ). The smaller (red) and larger (blue) circles denote Li and $\mathrm{N}$ ions, respectively. (b) and (c) Local relaxed geometries of the $\mathrm{Li}$ interstitials in two configurations $\left(\mathrm{Li}_{\mathrm{i}_{(1)}}\right.$ and $\mathrm{Li}_{\left.\mathrm{i}_{(2)}\right)}$, which are characterized by the Li dumbbells centered nearly at the $\operatorname{Li}(1)$ and $\operatorname{Li}(2)$ sites, respectively.

$$
\sum_{j} q_{j} C_{j}=0
$$

Using Eqs. (1), (5), and (6), the equilibrium concentrations of relevant defects, as well as the Fermi level and the formation energies, were determined under given atomic chemical potentials and temperature.

The energetically favorable migration paths of $\mathrm{Li}$ ions and their energy barriers were evaluated by dividing the paths connecting the configurations at the initial and final states into more than 15 hyperplanes. For each hyperplane, geometry optimization was performed with ionic relaxation restricted to the plane. This provides the lowest energy configuration within each hyperplane. The series of the relaxed ionic configurations and their energies constitute the trajectories and energy profiles of the Li ionic migration. The trajectories and energy profiles obtained in this way were confirmed to be smooth, as shown later in Sec. III C.

\section{RESULTS AND DISCUSSION}

\section{A. Defect species and equilibrium concentrations}

Figure 1(a) shows the unit cell of $\mathrm{Li}_{3} \mathrm{~N}$. The $\mathrm{Li}$ ions occupy the $\mathrm{Li}(1)$ and $\mathrm{Li}(2)$ sites in the $\mathrm{Li}$ and $\mathrm{Li}_{2} \mathrm{~N}$ layers, respectively. Vacancies at these sites were considered, which are referred to as $V_{\mathrm{Li}(1)}$ and $V_{\mathrm{Li}(2)}$. For $\mathrm{Li}_{\mathrm{i}}$, we found two configurations by geometry optimization. The local relaxed geometries are depicted in Figs. 1(b) and 1(c). Both are characterized by dumbbells, which consist of one $\mathrm{Li}$ ion from the lattice site and the other from the interstitial site. The dumbbells are centered nearly at the $\operatorname{Li}(1)$ and $\operatorname{Li}(2)$ sites and oriented parallel to the $a$ and $c$ axes, respectively. These configurations are denoted as $\mathrm{Li}_{\mathrm{i}_{(1)}}$ and $\mathrm{Li}_{\mathrm{i}_{(2)}}$.

The calculated equilibrium concentrations of native and H-related defects are shown in Fig. 2 for two extreme cases, i.e., undoped $\mathrm{Li}_{3} \mathrm{~N}$ at the $\mathrm{Li}$-rich limit and $\mathrm{H}$-doped $\mathrm{Li}_{3} \mathrm{~N}$ at the H-rich limit. The numbers of defect sites per unit cell, which were used for the evaluation of the concentrations, are one for $V_{\mathrm{Li}(1)}, \mathrm{Li}_{\mathrm{i}(1)}, V_{\mathrm{N}}, \mathrm{N}_{\mathrm{i}}, \mathrm{H}_{\mathrm{i}}$, and $\mathrm{H}_{\mathrm{Li}(1)}$ and two for $V_{\mathrm{Li}(2)}$, $\mathrm{Li}_{\mathrm{i}(2)}$, and $\mathrm{H}_{\mathrm{Li}(2)}$. In the undoped case presented in Fig. 2(a), only three types of defect, i.e., $V_{\mathrm{Li}(2)}^{-}, \mathrm{Li}_{\mathrm{i}(1)}^{+}$, and $\mathrm{Li}_{\mathrm{i}(2)}^{+}$, appear in the given concentration range; the other defects, i.e., $V_{\mathrm{Li}(1)}^{-}, V_{\mathrm{N}}^{3+}$, and $\mathrm{N}_{\mathrm{i}}^{3-}$, exhibit lower concentrations. We found that the $\mathrm{N}$-rich limit yields nearly identical concentration profiles with those for the Li-rich limit shown in Fig. 2(a). The defect concentrations are, thus, essentially independent 

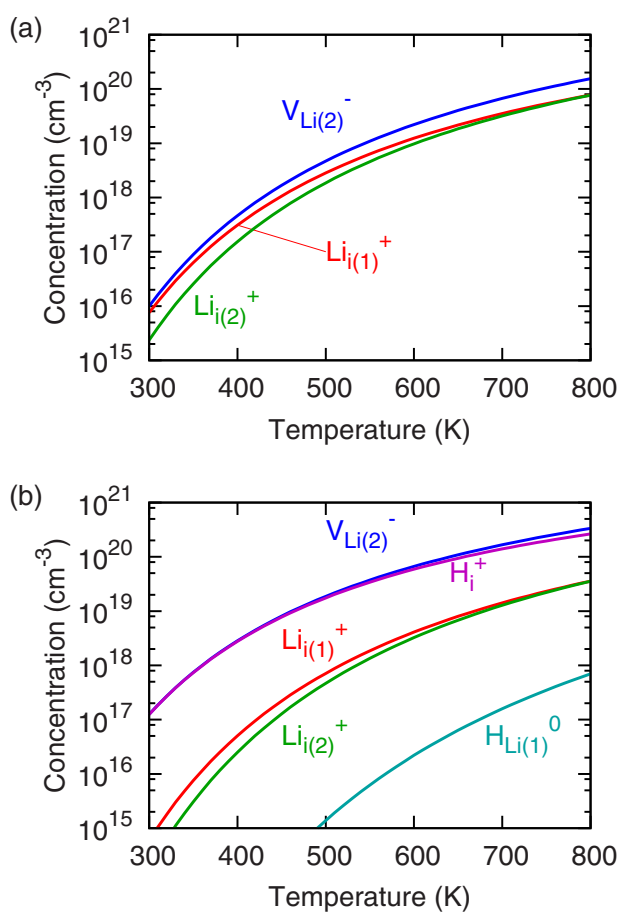

FIG. 2. (Color online) Equilibrium defect concentrations as a function of temperature under two chemical potential conditions: (a) undoped $\mathrm{Li}_{3} \mathrm{~N}$ at the Li-rich limit and (b) $\mathrm{H}$-doped $\mathrm{Li}_{3} \mathrm{~N}$ at the H-rich limit.

of $\mu_{\mathrm{Li}}$ and $\mu_{\mathrm{N}}$ in undoped $\mathrm{Li}_{3} \mathrm{~N}$. This is due to the defect equilibria dominated by two types of intrinsic Frenkel reaction as explained below.

At low temperatures $(\sim 300 \mathrm{~K})$, the predominant defects are $V_{\mathrm{Li}(2)}$ and $\mathrm{Li}_{\mathrm{i}(1)}^{+}$. They have nearly the same concentrations, indicating the dominance of the Frenkel reaction generating these defects. As the temperature increases, the concentration of $\mathrm{Li}_{\mathrm{i}(2)}^{+}$becomes closer to that of $\mathrm{Li}_{\mathrm{i}(1)}^{+}$. Therefore, another Frenkel reaction generating $V_{\mathrm{Li}(2)}$ and $\mathrm{Li}_{\mathrm{i}(2)}^{+}$also takes place at high temperatures. The charge neutrality condition requires that the concentration of $V_{\mathrm{Li}(2)}^{-}$is approximately equal to the sum of the concentrations of $\mathrm{Li}_{\mathrm{i}(1)}^{+}$and $\mathrm{Li}_{\mathrm{i}(2)}^{+}$. To maintain this condition, the chemical potential dependence of the formation energy of each defect is compensated by the change in the Fermi level in Eq. (1). This corresponds to the fact that the intrinsic Frenkel reaction does not depend on the chemical potentials.

Moving on to $\mathrm{H}$-doped $\mathrm{Li}_{3} \mathrm{~N}$ shown in Fig. 2(b), the defect equilibrium is altered from the undoped case. Under this chemical potential condition corresponding to the H-rich limit, we found that $\mathrm{H}_{\mathrm{Li}(2)}^{0}$ exhibits the highest concentration among the H-related defects. Its equilibrium concentration reaches the maximum value of $4.4 \times 10^{22} \mathrm{~cm}^{-3}$, for which all the $\mathrm{Li}(2)$ site is occupied by $\mathrm{H}$ [not shown in Fig. 2(b)]. This may result from the extreme $\mathrm{H}$-rich condition considered here. In addition, the concentration of $\mathrm{H}_{\mathrm{Li}(2)}^{0}$ may be overestimated because defect-defect interactions, which can be important for such high concentrations, are neglected in its evaluation using Eqs. (5) and (6). In the relaxed geometry of $\mathrm{H}_{\mathrm{Li}(2)}^{0}, \mathrm{H}$ is located near one of the nearest-neighbor $\mathrm{N}$ ions with a N-H distance of $1.04 \AA$. The formation of this
TABLE I. Formation energies of dominant native defects in undoped and $\mathrm{H}$-doped $\mathrm{Li}_{3} \mathrm{~N}$.

\begin{tabular}{lccc}
\hline \hline & \multicolumn{3}{c}{ Formation energy $(\mathrm{eV})$} \\
& $V_{\mathrm{Li}(2)}$ & $\mathrm{Li}_{\mathrm{i}(1)}^{+}$ & $\mathrm{Li}_{\mathrm{i}(2)}^{+}$ \\
\hline Undoped & 0.40 & 0.39 & 0.43 \\
$\mathrm{H}$ doped & 0.33 & 0.45 & 0.50 \\
\hline \hline
\end{tabular}

$\mathrm{NH}^{2-}$-like configuration is consistent with a previous experimental report. ${ }^{6}$ In terms of the $\mathrm{Li}$ ionic conduction, however, this defect is not directly relevant because its neutral charge state does not affect the concentrations of the charge carriers, i.e., charged $\mathrm{Li}$ defects; instead, $\mathrm{H}_{\mathrm{i}}^{+}$plays an important role. As shown in Fig. 2(b), the charge neutrality condition is met mostly by the equalization of the concentrations of $\mathrm{H}_{\mathrm{i}}^{+}$and $V_{\mathrm{Li}(2)}$. In contrast to the undoped case, $\mathrm{Li}_{\mathrm{i}(1)}^{+}$and $\mathrm{Li}_{\mathrm{i}(2)}^{+}$have one to two orders of magnitude smaller concentrations than $V_{\mathrm{Li}(2)}^{-}$.

\section{B. Defect formation energies}

To discuss the formation energy contribution to the activation energy for the $\mathrm{Li}$ ionic conduction, the formation energies of $V_{\mathrm{Li}}^{-}$and $\mathrm{Li}_{\mathrm{i}}^{+}$were evaluated. The results are shown in Table I. In the undoped case, the formation energies were obtained at the Li-rich limit and $T=300 \mathrm{~K}$, using Eq. (1) and the Fermi level determined via the charge neutrality condition given by Eq. (6). It is noted, however, that the values are almost independent of the $\mathrm{Li}$ and $\mathrm{N}$ chemical potentials, corresponding to the behavior of the equilibrium concentrations as mentioned in Sec. III A. In addition, the formation energies are essentially independent of temperature; the largest variation is only $0.01 \mathrm{eV}$ in the temperature range from 300 to $700 \mathrm{~K}$, which is typical for the ionic conductivity measurement for $\mathrm{Li}_{3} \mathrm{~N}$.

The formation energies of $V_{\mathrm{Li}(2)}, \mathrm{Li}_{\mathrm{i}(1)}^{+}$, and $\mathrm{Li}_{\mathrm{i}(2)}^{+}$are close to each other in the undoped case. This is expected from their similar concentrations shown in Fig. 2(a); the difference in the number of defect sites by a factor of 2 exerts small influences. Meanwhile, the formation energy of $V_{\mathrm{Li}(1)}$ (not shown in Table I) is $1.77 \mathrm{eV}$ higher than that of $V_{\mathrm{Li}(2)}$. This is consistent with the results of previously reported firstprinciples calculations ${ }^{14}$ and atomistic simulations. ${ }^{11}$

For $\mathrm{H}$-doped $\mathrm{Li}_{3} \mathrm{~N}$, the formation energy contribution to the $\mathrm{Li}$ ionic conduction can be estimated by assuming likely extrinsic conditions, which are given as follows: (i) the $\mathrm{H}$ concentration is constant during conductivity measurements with varying temperature and (ii) $V_{\mathrm{Li}(2)}$ is mainly generated via the dissociation of $\mathrm{H}_{\mathrm{Li}(2)}^{0}$, which is the dominant $\mathrm{H}$-related defect as mentioned in Sec. III A, into $\mathrm{H}_{\mathrm{i}}^{+}$and $V_{\mathrm{Li}(2)}^{-}$. Whether the latter condition holds or not is determined by the competition between the formation of $V_{\mathrm{Li}(2)}$ via the dissociation of $\mathrm{H}_{\mathrm{Li}(2)}^{0}$ and that via the intrinsic Frenkel reactions, which are, respectively, given as

$$
\mathrm{H}_{\mathrm{Li}(2)}^{0} \rightarrow V_{\mathrm{Li}(2)}+\mathrm{H}_{\mathrm{i}}^{+}, \quad \frac{\left[V_{\mathrm{Li}(2)}\right]\left[\mathrm{H}_{\mathrm{i}}^{+}\right]}{\left[\mathrm{H}_{\mathrm{Li}(2)}^{0}\right]}=2 \exp \left(-\frac{E_{1}}{k_{\mathrm{B}} T}\right),
$$




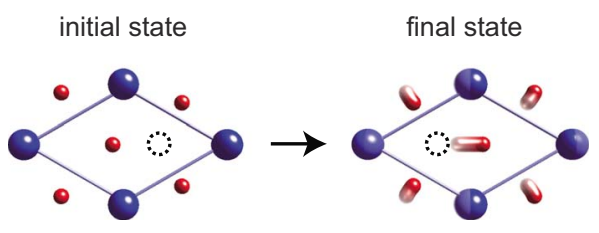

FIG. 3. (Color online) Predicted mechanism for the intralayer migration of $V_{\mathrm{Li}}$. The smaller (red) and larger (blue) circles denote $\mathrm{Li}$ and $\mathrm{N}$ ions, respectively: the filled circles for the relaxed geometries at the initial and final states around $V_{\mathrm{Li}(2)}$ and the translucent circles for the trajectories of ionic motions. The $V_{\mathrm{Li}(2)}^{-}$positions at the initial and final states are designated by the dotted circles.

$$
\begin{aligned}
& \text { null } \rightarrow V_{\mathrm{Li}(2)}^{-}+\mathrm{Li}_{\mathrm{i}(1)}^{+}, \quad\left[V_{\mathrm{Li}(2)}^{-}\right]\left[\mathrm{Li}_{\mathrm{i}(1)}^{+}\right]=2 \exp \left(-\frac{E_{2}}{k_{\mathrm{B}} T}\right), \\
& \text { null } \rightarrow V_{\mathrm{Li}(2)}^{-}+\mathrm{Li}_{\mathrm{i}(2)}^{+}, \quad\left[V_{\mathrm{Li}(2)}^{-}\right]\left[\mathrm{Li}_{\mathrm{i}(2)}^{+}\right]=4 \exp \left(-\frac{E_{3}}{k_{\mathrm{B}} T}\right),
\end{aligned}
$$

where $[j]$ denotes the concentration of defect $j$, i.e., $C_{j}$ in Eq. (5). $E_{1}=0.66 \mathrm{eV}, E_{2}=0.78 \mathrm{eV}$, and $E_{3}=0.83 \mathrm{eV}$ were obtained using the calculated formation energies for constituent defects in these chemical equations. Note that the values of $E_{1}, E_{2}$, and $E_{3}$ are independent of the temperature. We assume $\left[\mathrm{H}_{\mathrm{Li}(2)}^{0}\right]$ to be a constant since $\left[\mathrm{H}_{\mathrm{Li}(2)}^{0}\right] \gg\left[\mathrm{H}_{\mathrm{i}}^{+}\right]$holds for $T<700 \mathrm{~K}$ as mentioned in Sec. III A and hence the total $\mathrm{H}$ concentration, which is assumed to be a constant, is nearly equal to $\left[\mathrm{H}_{\mathrm{Li}(2)}^{0}\right]$. The $\mathrm{H}$ dissociation reaction given by Eq. (7) was found to dominate over the Frenkel reactions given by Eqs. (8) and (9) when the $\mathrm{H}$ concentration is higher than $\sim 1 \%$ at $300 \mathrm{~K}(\sim 10 \%$ at $700 \mathrm{~K})$. In this extrinsic regime, the charge neutrality condition is approximately given as

$$
\left[V_{\mathrm{Li}(2)}\right]=\left[\mathrm{H}_{\mathrm{i}}^{+}\right] .
$$

Equations (7) and (10) yield the formation energy of $V_{\mathrm{Li}(2)}$ as $1 / 2 E_{1}=0.33 \mathrm{eV}$. The formation energies of $\mathrm{Li}_{\mathrm{i}(1)}^{+}$and $\mathrm{Li}_{\mathrm{i}(2)}^{+}$ are then obtained using Eqs. (8) and (9) as $E_{2}-1 / 2 E_{1}$ $=0.45 \mathrm{eV}$ and $E_{3}-1 / 2 E_{1}=0.50 \mathrm{eV}$, respectively. As shown in Table I, the resultant formation energy of $V_{\mathrm{Li}(2)}$ is lower and those of $\mathrm{Li}_{\mathrm{i}(1)}^{+}$and $\mathrm{Li}_{\mathrm{i}(2)}^{+}$are higher than the corresponding undoped values. Thus, the incorporation of $\mathrm{H}$ impurities changes the defect equilibria and hence the formation energies of the native defects. As discussed later, this affects the activation energy for the $\mathrm{Li}$ ionic conduction in $\mathrm{Li}_{3} \mathrm{~N}$.

\section{Migration paths and energies of $\mathrm{Li}$ ions}

The migration paths and energies of $V_{\mathrm{Li}}$ and $\mathrm{Li}_{\mathrm{i}}$ were determined for the intralayer and interlayer directions. The results are shown in Figs. 3-5. Concerning the migration of $V_{\mathrm{Li}}$, we focus on $V_{\mathrm{Li}(2)}$ because $V_{\mathrm{Li}(1)}^{-}$with a $1.8 \mathrm{eV}$ higher formation energy is expected to make a negligible contribution. The migration of $V_{\mathrm{Li}(2)}$ via $V_{\mathrm{Li}(1)}$ as an intermediate state is unlikely for the same reason. In the case of the intralayer migration, it was found that one of the $\operatorname{Li}(2)$ ions adjacent to $V_{\mathrm{Li}(2)}$ on the same $\mathrm{Li}_{2} \mathrm{~N}$ layer simply moves to the (a)

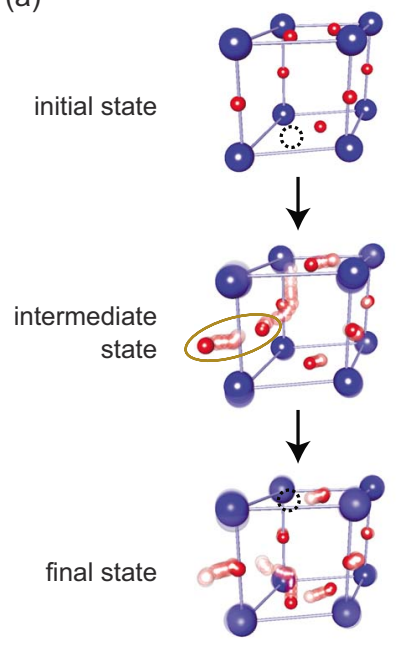

(b)

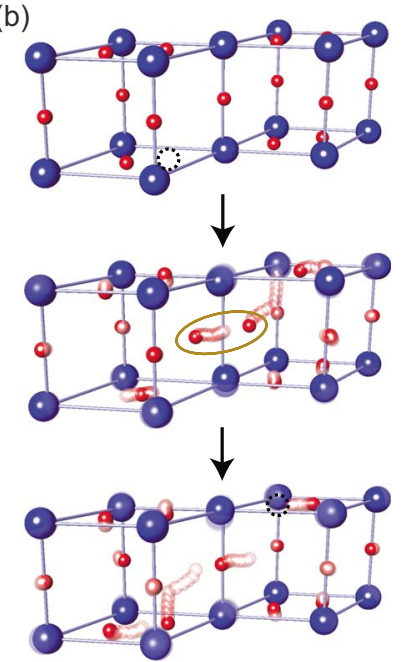

(c)

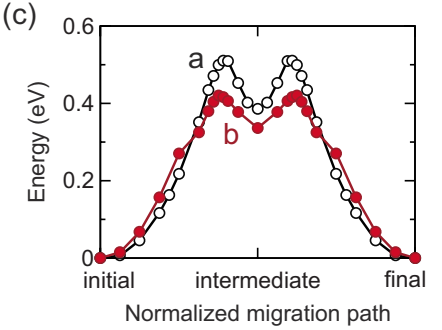

FIG. 4. (Color online) Predicted mechanisms for the interlayer migration of $V_{\mathrm{Li}}$. (a) Migration toward the $\mathrm{Li}(2)$ site located above the initial $V_{\mathrm{Li}(2)}^{-}$position. (b) Toward the $\mathrm{Li}(2)$ site in another unit cell. Relaxed geometries and ionic motions are presented in the same manner as Fig. 3. Dumbbell-like structures at the intermediate states are enclosed. (c) Energy profiles for the migration paths shown in (a) and (b).

vacancy site as shown in Fig. 3. The highest barrier height for the migration, i.e., the migration energy, was estimated to be only $0.01 \mathrm{eV}$. This value is close to those predicted in previous first-principles studies. ${ }^{14,15}$

In contrast to the intralayer migration, the migration of $V_{\mathrm{Li}(2)}^{-}$in the interlayer direction was found to occur via metastable configurations at the intermediate states. Among the migration paths obtained through extensive searches, two typical paths with low migration energies are presented in Fig. 4. In these paths, $V_{\mathrm{Li}(2)}$ migrates via similar transient dumbbell-like configurations at the $\operatorname{Li}(1)$ sites but toward different $\mathrm{Li}(2)$ sites. In the path indicated in Fig. 4(a) [path (a)], $V_{\mathrm{Li}(2)}^{-}$migrates toward the $\mathrm{Li}(2)$ site located above the initial $V_{\mathrm{Li}(2)}$ position. On the other hand, $V_{\mathrm{Li}(2)}$ migrates from one unit cell to another in path (b), yielding a lower migration energy (barrier height for the migration) than path (a) as shown in Fig. 4(c). This migration mechanism has been previously suggested by Sarnthein et al. ${ }^{14}$ through firstprinciples calculations focused on the $V_{\mathrm{Li}}$ migration. The present migration energy of $0.42 \mathrm{eV}$ is $0.16 \mathrm{eV}$ lower than the reported value. This may be due to the use of larger supercells in the present calculations, where more ions can relax. In the two studies, the following common important conclusions have been drawn: (i) the most favorable path for 


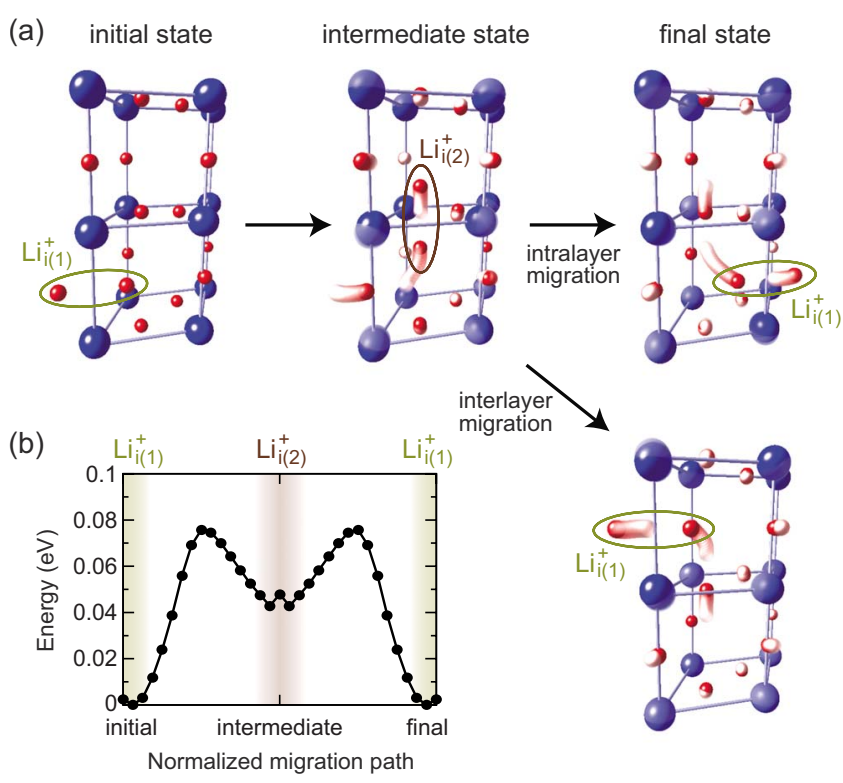

FIG. 5. (Color online) Predicted mechanism for the intralayer and interlayer migrations of $\mathrm{Li}_{\mathrm{i}}$. (a) Relaxed geometries and ionic motions, which are presented in the same manner as Figs. 3 and 4. (b) Energy profile. Mirror symmetric configurations for $\mathrm{Li}_{\mathrm{i}(1)}^{+}$and $\mathrm{Li}_{\mathrm{i}(2)}^{+}$are taken as the left and right edges and the center of the abscissa representing the normalized migration path, respectively.

the interlayer migration of $V_{\mathrm{Li}}$ is path (b) and (ii) the energy barrier for the interlayer migration is at least $0.4 \mathrm{eV}$ higher than that for the intralayer migration.

The predicted path and energy profile of $\mathrm{Li}_{\mathrm{i}}$ migration with the lowest energy barrier are presented in Fig. 5. The migration mechanism involves both $\mathrm{Li}_{\mathrm{i}(1)}^{+}$and $\mathrm{Li}_{\mathrm{i}(2)}^{+}$: taking $\mathrm{Li}_{\mathrm{i}(1)}^{+}$as the initial configuration, one of the $\mathrm{Li}$ ions in the $\mathrm{Li}_{\mathrm{i}(1)}^{+}$dumbbell moves toward the $\mathrm{Li}(2)$ site to form a $\mathrm{Li}_{\mathrm{i}(2)}^{+}$ dumbbell, and then, the $\mathrm{Li}_{\mathrm{i}(2)}^{+}$dumbbell turns into a $\mathrm{Li}_{\mathrm{i}(1)}^{+}$ dumbbell, which is located on the right-hand side of the initial configuration (intralayer migration) or above (interlayer migration) in Fig. 5(a). For both $\mathrm{Li}_{\mathrm{i}(1)}^{+}$and $\mathrm{Li}_{\mathrm{i}(2)}^{+}$, the dumbbells in their lowest energy configurations are slightly off centered from the $\mathrm{Li}(1)$ and $\mathrm{Li}(2)$ sites, i.e., not mirror symmetric, but the energy differences from the symmetric configurations are marginal $(\sim 0.01 \mathrm{eV})$, as shown in Fig. 5(b). In other words, the potential is nearly flat around the symmetric configurations. The highest energy barrier for the migration is located around the middle of the $\mathrm{Li}_{\mathrm{i}(1)}^{+}$and $\mathrm{Li}_{\mathrm{i}(2)}^{+}$ configurations. It is noteworthy that the barrier height is only $0.08 \mathrm{eV}$ and that this mechanism is common to the intralayer and interlayer migrations, indicating that $\mathrm{Li}_{\mathrm{i}}$ is mobile in both directions.

\section{Activation energies for $\mathrm{Li}$ ionic conduction}

The activation energies for the $\mathrm{Li}$ ionic conduction were evaluated as the sum of the formation and migration energies. The results are summarized in Table II. For $V_{\mathrm{Li}}$, the activation energies of $V_{\mathrm{Li}(2)}^{-}$having a much higher concentration (and also much lower formation energy) than $V_{\mathrm{Li}(1)}$ are taken. For $\mathrm{Li}_{i}$, since $\mathrm{Li}_{\mathrm{i}(1)}^{+}$and $\mathrm{Li}_{i(2)}^{+}$are very similar in the
TABLE II. Activation energies for the intralayer and interlayer conductions of $V_{\mathrm{Li}}$ and $\mathrm{Li}_{\mathrm{i}}$ in undoped and $\mathrm{H}$-doped $\mathrm{Li}_{3} \mathrm{~N}$, along with experimental activation energies for the $\mathrm{Li}$ ionic conduction (Refs. 6 and 7) and diffusion (Refs. 2 and 4). Values for the charge carriers that are likely to make minor contributions are shown in parentheses (see text for the details).

\begin{tabular}{|c|c|c|c|c|}
\hline & \multicolumn{4}{|c|}{ Activation energy $(\mathrm{eV})$} \\
\hline & \multicolumn{2}{|c|}{ Undoped } & \multicolumn{2}{|c|}{$\mathrm{H}$ doped } \\
\hline & Intralayer & Interlayer & Intralayer & Interlayer \\
\hline$V_{\mathrm{Li}}$ & 0.40 & $(0.81)$ & 0.34 & 0.75 \\
\hline $\mathrm{Li}_{\mathrm{i}}$ & 0.47 & 0.47 & $(0.53)$ & 0.53 \\
\hline Expt. $^{\mathrm{a}}$ & 0.56 & 0.67 & 0.28 & 0.59 \\
\hline Expt. $^{b}$ & & & $0.23,0.25$ & $0.65,0.78$ \\
\hline Expt. $^{\mathrm{c}}$ & 0.41 & 0.68 & & \\
\hline Expt. $^{\mathrm{d}}$ & & 0.65 & & \\
\hline
\end{tabular}

${ }^{\text {aReference } 6 .}$

${ }^{\mathrm{b}}$ Reference 7.

${ }^{\mathrm{c}}$ Reference 4.

${ }^{\mathrm{d}}$ Reference 2 .

formation energy and both of them are involved in the migration process as described above, $\mathrm{Li}_{\mathrm{i}(1)}^{+}$and $\mathrm{Li}_{\mathrm{i}(2)}^{+}$are denoted together as $\mathrm{Li}_{\mathrm{i}(1,2)}^{+}$; for simplicity, the formation energy of $\mathrm{Li}_{\mathrm{i}(1)}^{+}$was employed for that of $\mathrm{Li}_{\mathrm{i}(1,2)}^{+}$.

In the case of undoped $\mathrm{Li}_{3} \mathrm{~N}$, in which the defect equilibrium is governed by the Frenkel reactions, the concentrations of the two dominant defects, i.e., $V_{\mathrm{Li}(2)}$ and $\mathrm{Li}_{\mathrm{i}(1,2)}^{+}$, are approximately equal, resulting in the approximately equal formation energies, as discussed in Sec. III A. Therefore, the difference in activation energy is given by the migration energy contributions. In view of the resultant activation energy, $V_{\mathrm{Li}(2)}^{-}$is slightly preferable to $\mathrm{Li}_{\mathrm{i}(1,2)}^{+}$for the intralayer conduction, whereas $\mathrm{Li}_{\mathrm{i}(1,2)}^{+}$is much more favorable for the interlayer conduction and hence $V_{\mathrm{Li}(2)}$ is expected to make a minor contribution. Notably, the orientation dependence of the lowest activation energy is very small despite the anisotropic layered structure and the different charge carriers between the intralayer and interlayer directions. It is also noted that the concentrations of the two types of charge carrier are nearly the same.

For $\mathrm{H}$-doped $\mathrm{Li}_{3} \mathrm{~N}$, the activation energy of $V_{\mathrm{Li}(2)}$ decreases from the undoped value owing to the decrease in its formation energy, while the formation energy of $\mathrm{Li}_{i(1,2)}^{+}$and hence its activation energy increases, as discussed in Sec. III B. $V_{\mathrm{Li}(2)}^{-}$with a higher concentration and a lower activation energy is expected to dominate the intralayer conduction. For the interlayer conduction, either $V_{\mathrm{Li}(2)}^{-}$with a higher concentration and a higher activation energy or $\mathrm{Li}_{i(1,2)}^{+}$with a lower concentration and a lower activation energy can be the major carrier, depending on the $\mathrm{H}$ concentration in the specimen and the conductivity measurement temperature. In both cases, particularly in the former, the anisotropy is obvious compared to the undoped system. The present results thus suggest that the anisotropic conduction and diffusion of $\mathrm{Li}$ ions in $\mathrm{Li}_{3} \mathrm{~N}$ are induced by the $\mathrm{H}$ incorporation. For comparison, experimental activation energies for the $\mathrm{Li}$ ionic 
conduction $^{6,7}$ and diffusion ${ }^{2,4}$ are also listed in Table II. The experimental results show a dispersion, but a tendency that the H-doped specimens exhibit a larger anisotropy is recognized. The calculated activation energies are close to the experimental values. More notably, they reproduce the variable anisotropic behavior associated with the $\mathrm{H}$ incorporation.

\section{CONCLUSIONS}

The formation and migration of $V_{\mathrm{Li}}$ and $\mathrm{Li}_{\mathrm{i}}$ in $\mathrm{Li}_{3} \mathrm{~N}$ have been investigated using first-principles calculations, including the effect of the $\mathrm{H}$ impurity. Contrary to previous understanding, we found that $\mathrm{Li}_{\mathrm{i}}$ forms in two types of dumbbell structure with low formation energies and that $\mathrm{Li}_{i}$ in these structures readily migrates and hence plays an essential role in the Li ionic conduction as well as $V_{\mathrm{Li}}$. Despite the anisotropic layered structure of $\mathrm{Li}_{3} \mathrm{~N}$, the $\mathrm{Li}$ ionic conduction is nearly isotropic in the undoped system. This behavior is attributed to the dominance of the Frenkel reactions generating $V_{\mathrm{Li}}$ and $\mathrm{Li}_{\mathrm{i}}$, which results in nearly equal formation energies of the two charge carriers, and to the low migration energies for $V_{\mathrm{Li}}$ in the intralayer direction and for $\mathrm{Li}_{\mathrm{i}}$ in both direc- tions. Under the presence of $\mathrm{H}$ impurities, an anisotropy in the ionic conduction arises from $V_{\mathrm{Li}}$-rich defect equilibria, which enhance and suppress the formation of $V_{\mathrm{Li}}$ and $\mathrm{Li}_{\mathrm{i}}$, respectively. Our results show that even such a prototypical ionic conductor as $\mathrm{Li}_{3} \mathrm{~N}$ exhibits a unique conduction mechanism including the presence of duplex-charge carriers, their easy motions via metastable intermediate configurations, and the $\mathrm{H}$-induced change in defect equilibrium, which leads to its anisotropic conductivity. Since similar mechanisms can hold for other materials, these insights would renew interest in pre-existing ionic conductors. They also provide a useful guideline for designing a new class of high-performance ionic conductors.

\section{ACKNOWLEDGMENTS}

This work was supported by the Grants-in-Aid for Scientific Research (A) and Priority Areas "Atomic Scale Modification" (No. 474) and the Global COE Program "International Center for Integrated Research and Advanced Education in Materials Science," all from the Ministry of Education, Culture, Sports, Science and Technology of Japan.
*Present address: Department of Intelligent Materials Engineering, Osaka City University, Sumiyoshi, Osaka 558-8585, Japan; kishida@imat.eng.osaka-cu.ac.jp

†oba@cms.mtl.kyoto-u.ac.jp

${ }^{1}$ D. Brinkmann, M. Mali, J. Roos, R. Messer, and H. Birli, Phys. Rev. B 26, 4810 (1982).

${ }^{2}$ M. Wilkening, D. Gebauer, and P. Heitjans, J. Phys.: Condens. Matter 20, 022201 (2008).

${ }^{3}$ B. Bader, P. Heitjans, H. J. Stöckmann, H. Ackermann, W. Buttler, P. Freiländer, G. Kiese, C. Vandermarel, and A. Schirmer, J. Phys.: Condens. Matter 4, 4779 (1992).

${ }^{4}$ E. Bechtold-Schweickert, M. Mali, J. Roos, and D. Brinkmann, Phys. Rev. B 30, 2891 (1984).

${ }^{5}$ H. Schulz and K. H. Thiemann, Acta Crystallogr., Sect. A: Cryst. Phys., Diffr., Theor. Gen. Crystallogr. 35, 309 (1979).

${ }^{6}$ J. Wahl, Solid State Commun. 29, 485 (1979).

${ }^{7}$ A. Hooper, T. Lapp, and S. Skaarup, Mater. Res. Bull. 14, 1617 (1979).

${ }^{8}$ U. v. Alpen, A. Rabenau, and G. H. Talat, Appl. Phys. Lett. 30, 621 (1977).

${ }^{9}$ G. Kerker, Phys. Rev. B 23, 6312 (1981).

${ }^{10}$ P. Blaha, K. Schwarz, and P. Herzig, Phys. Rev. Lett. 54, 1192 (1985).

${ }^{11}$ J. R. Walker and C. R. A. Catlow, Philos. Mag. A 43, 265 (1981).

${ }^{12}$ M. L. Wolf, J. R. Walker, and C. R. A. Catlow, J. Phys. C 17, 6623 (1984)

${ }^{13}$ M. L. Wolf and C. R. A. Catlow, J. Phys. C 17, 6635 (1984).

${ }^{14}$ J. Sarnthein, K. Schwarz, and P. E. Blöchl, Phys. Rev. B 53, 9084 (1996).
${ }^{15}$ Y. Koyama, Y. Yamada, I. Tanaka, S. R. Nishitani, H. Adachi, M. Murayama, and R. Kanno, Mater. Trans. 43, 1460 (2002).

${ }^{16}$ Z. Stoeva, R. Gomez, A. G. Gordon, M. Allan, D. H. Gregory, G. B. Hix, and J. J. Titman, J. Am. Chem. Soc. 126, 4066 (2004).

${ }^{17}$ J. Rowsell, V. Pralong, and L. Nazar, J. Am. Chem. Soc. 123, 8598 (2001).

${ }^{18}$ P. Chen, Z. T. Xiong, J. Z. Luo, J. Y. Lin, and K. L. Tan, Nature (London) 420, 302 (2002).

${ }^{19}$ M. Nishijima, T. Kagohashi, M. Imanishi, Y. Takeda, O. Yamamoto, and S. Kondo, Solid State Ionics 83, 107 (1996).

${ }^{20}$ P. Hohenberg and W. Kohn, Phys. Rev. 136, B864 (1964).

${ }^{21}$ W. Kohn and L. J. Sham, Phys. Rev. 140, A1133 (1965).

${ }^{22}$ P. E. Blöchl, Phys. Rev. B 50, 17953 (1994).

${ }^{23}$ G. Kresse and J. Hafner, Phys. Rev. B 48, 13115 (1993).

${ }^{24}$ G. Kresse and J. Furthmüller, Phys. Rev. B 54, 11169 (1996).

${ }^{25}$ G. Kresse and D. Joubert, Phys. Rev. B 59, 1758 (1999).

${ }^{26}$ J. P. Perdew, K. Burke, and M. Ernzerhof, Phys. Rev. Lett. 77, 3865 (1996).

${ }^{27}$ H. Schulz and K. Schwarz, Acta Crystallogr., Sect. A: Cryst. Phys., Diffr., Theor. Gen. Crystallogr. 34, 999 (1978).

${ }^{28}$ S. Pöykkö, M. J. Puska, and R. M. Nieminen, Phys. Rev. B 53, 3813 (1996).

${ }^{29}$ C. G. Van de Walle and J. Neugebauer, J. Appl. Phys. 95, 3851 (2004).

${ }^{30}$ A. Togo, F. Oba, I. Tanaka, and K. Tatsumi, Phys. Rev. B 74, 195128 (2006).

${ }^{31}$ M. Choi, F. Oba, and I. Tanaka, Phys. Rev. B 78, 014115 (2008).

${ }^{32}$ J. Maier, Physical Chemistry of Ionic Materials: Ions and Electrons in Solids (Wiley, New York, 2004). 